\title{
Diagnóstico de Trastorno del Espectro Autista- TEA, adaptándonos a la nueva realidad,
}

\section{Telesalud.}

\author{
Diagnosis of Autistic Spectrum Disorder-ASD, adapting to the new reality, Telehealth. \\ Myriam Velarde-Incháustegui ${ }^{1,2,3, a}$, María Elena Ignacio-Espíritu ${ }^{3,4, b}$, Aland Cárdenas-Soza ${ }^{3,5, c}$ \\ RESUMEN
}

Los Trastornos del Espectro Autista (TEA) son un grupo de alteraciones del neurodesarrollo que afectan la comunicación y el comportamiento. El Centro de Control de Enfermedades (CDC, siglas en inglés) señaló que la prevalencia de TEA en Estados Unidos, hacia el año 2016, era de 1 de cada 54 niños menores de 8 años. Los criterios de diagnóstico clínico se encuentran tanto en el Manual Diagnóstico y Estadístico de Trastornos Mentales $5^{\text {ta }}$ edición (DSM - 5) como en la Clasificación Internacional de Enfermedades, 10 ${ }^{\text {ma }}$ edición (CIE-10). Las herramientas de ayuda diagnóstica más usadas y que poseen un alto grado de confiabilidad para el cribado del TEA son las entrevistas semiestructuradas y cuestionarios como la Lista de Verificación Modificada para el Autismo en niños pequeños (M-CHAT), la Entrevista para el Diagnóstico del Autismo - Revisada (ADI-R), y la Escala de Observación para el Diagnóstico del Autismo (ADOS). Entre las nuevas herramientas debe mencionarse el uso combinado de la Preferencia de Mirada y el M-CHAT-R (GP-M-CHAT/R). Por su parte, Telesalud es un servicio que utiliza herramientas como Tecnologías de Información y Comunicaciones (TIC), cuya viabilidad, accesibilidad y confiabilidad para el diagnóstico de trastornos del neurodesarrollo como TEA, se vienen estudiando con resultados satisfactorios en diversas partes del mundo.

PALABRAS CLAVE: Trastornos del Espectro Autista (TEA), diagnóstico, Telesalud, detección, trastornos del neurodesarrollo, seguimiento ocular.

\section{SUMMARY}

Autism Spectrum Disorders (ASD) are a group of neurodevelopmental disorders that affect communication and behavior. The Centers for Disease Control and Prevention (CDC) pointed out on March 30, 2020, that the prevalence of ASD in the United States in 2016 was of 1 in 54 children under the age of 8 . The clinical diagnostic criteria are found in both DSM-5 and ICD-10. Diagnostic aid tools used for ASD screening include semi-structured interviews and questionnaires such as the Modified Checklist for Autism in Young Children (M-CHAT), the Autism Diagnosis Interview - Revised (ADI-R), and the Autism Diagnosis Observation Scale (ADOS), all widely used and with a high degree of reliability. Among the new tools, it is worth to mention the combined use of gaze

\footnotetext{
Instituto Nacional de Ciencias Neurológicas. Lima, Perú.

Facultad de Medicina, Universidad Nacional Mayor de San Marcos. Lima, Perú.

Capítulo del Neurodesarrollo, Sociedad Peruana de Neurología. Lima, Perú.

EsSalud-Red Almenara. Lima, Perú.

Hospital de Apoyo de Aplao. Arequipa, Perú.

Magister en Educación; Médico Neurólogo; ID ORCID: 0000-0002-9977-4377/

Médico Neurólogo; ID ORCID: 0000-0001-8027-2599

Médico Neurólogo; ID ORCID 0000-0002-6847-5737
} 
preference and the M-CHAT-R (GP-M-CHAT / R). On its side, Telehealth is a health service that uses tools such as Information and Communication Technologies (ICT) whose viability, accessibility and reliability for the diagnosis of neurodevelopmental disorders such as ASD has been studied in different parts of the world, with satisfactory results.

KEYWORDS: Autism Spectrum Disorders (ASD), diagnosis, Telehealth, detection, developmental disorder, eye tracking.

\section{INTRODUCCIÓN}

El Trastorno del Espectro Autista (TEA) es una condición que amerita un diagnóstico temprano, que resulta muy difícil dado los altos costos económicos, tiempo de espera y limitado número de profesionales especializados. Sumado a ello tenemos al actual contexto socio sanitario debido a la pandemia Covid-19, lo que complica aún más la evaluación médica, siendo fundamental conocer qué alternativas se han desarrollado para la detección y diagnóstico en este contexto.

El TEA es un grupo de las alteraciones del neurodesarrollo que afecta la comunicación y el comportamiento $(1,2)$. En los Estados Unidos, según el Centro de Control de Enfermedades (CDC), la prevalencia del TEA en el año 2000 fue 1 de cada 154 niños menores de 8 años $(0,6 \%)$ y al 2016 fue 1 de cada 54, con una frecuencia de 4.3 veces mayor en niños que niñas $(1,2)$. México reporta que 1 de cada 115 niños tienen TEA (3). En Colombia, el $16 \%$ de los menores de 15 años cursan con el diagnóstico de Trastorno del Desarrollo, no teniendo cifras exclusivas del TEA (4). En Ecuador, se estima una prevalencia de $0,28 \%$ en niños menores de 5 años (5).

En el Perú, el Registro del Consejo Nacional para la Integración de la Persona con Discapacidad (CONADIS), no cuenta con datos de prevalencia del TEA a nivel nacional. Sin embargo, al 31 de agosto del 2018 , reporta inscritas $4528(2, .06 \%)$ con diagnóstico del TEA (80,9\% varones y $19,1 \%$ mujeres), con una tendencia de incremento anual (6).

Los datos obtenidos respecto al número de casos con el diagnóstico del TEA, denotan un claro incremento de la prevalencia de esta condición.

\section{Diagnóstico}

El diagnóstico temprano del TEA permite iniciar un tratamiento intensivo prontamente y lograr un efecto positivo en el neurodesarrollo del niño $(1,7,8)$.
Realizar el diagnóstico del TEA es complejo, por su particularidad, en cómo se manifiesta y cómo se expresan los síntomas a lo largo de las diferentes etapas de la vida, con el añadido de no existir un biomarcador específico que determine el trastorno $(3,9)$; siendo el diagnóstico fundamentalmente clínico. Se inicia con las personas que comparten el día a día con el niño, como los padres y profesores, quienes lo derivan al área de crecimiento y desarrollo en el primer nivel de atención, de existir criterios positivos los referirán al médico especialista (neurólogo del neurodesarrollo, neuropediatra, psiquiatra infantil ) para determinar el diagnóstico y solicitar las evaluaciones necesarias al equipo interdisciplinario (psicólogo, terapeuta ocupacional, terapeuta del aprendizaje, terapeuta físico y terapeuta de lenguaje) $(7,8,9)$.

Los criterios de diagnóstico clínico del TEA se encuentran en el Manual Diagnóstico y Estadístico de los Trastornos Mentales 5ta edición (DSM-5) y en la Clasificación Internacional de Enfermedades 10ma edición (CIE-10), con adaptaciones en su edición CIE11 (World Health Organization, 2018) que entrará en vigencia el 1 de enero del 2022, incorporando el término "Trastorno del Espectro Autista" $(10,11)$.

Los criterios diagnósticos considerados en el DSM 5 del TEA, se organizan en dos dimensiones centrales; déficit en la comunicación e interacción social y patrones de comportamientos restringidos, repetitivos y estereotipados, que los acompañan durante toda su vida de manera variable (tabla 1$)(8,9,10)$. Asimismo, establece tres niveles de gravedad fundamentado en los déficits de las dos dimensiones en que se organizan los criterios determinando el nivel de apoyo necesario para el planteamiento de la intervención y nivel de discapacidad que se debe indicar en el diagnóstico (10) (tabla 1).

\section{Situación actual de detección del TEA en Latinoamérica}

Las investigaciones han permitido precisar los primeros indicadores de TEA en el primer año de 
Tabla 1. Criterios diagnósticos del Trastorno del espectro autista y niveles de gravedad

Criterios diagnósticos

A. Deficiencias persistentes en la comunicación social y en la interacción social en diversos contextos

B. Patrones restrictivos y repetitivos de comportamiento, intereses o actividades

C. Los síntomas han de estar presentes en las primeras fases del período de desarrollo.

D. Los síntomas causan un deterioro clínicamente significativo en lo social, laboral u otras áreas importantes del funcionamiento habitual.

E. Estas alteraciones no se explican mejor por la discapacidad intelectual (trastorno del desarrollo intelectual) o por el retraso global del desarrollo.

Niveles de gravedad:

\begin{tabular}{|c|c|c|}
\hline & Comunicación social & $\begin{array}{l}\text { Comportamientos restringidos y } \\
\text { repetitivos }\end{array}$ \\
\hline $\begin{array}{l}\text { Grado } 3 \\
\text { "Necesita ayuda } \\
\text { muy notable" }\end{array}$ & $\begin{array}{l}\text { Deficiencias graves de las aptitudes de } \\
\text { comunicación social verbal y no verbal causan } \\
\text { alteraciones graves del funcionamiento, inicio muy } \\
\text { limitado de las interacciones sociales y respuesta } \\
\text { mínima a la apertura social de otras personas. }\end{array}$ & $\begin{array}{l}\text { La inflexibilidad extrema de } \\
\text { comportamiento, u otros comportamientos } \\
\text { restringidos/repetitivos Ansiedad intensa/ } \\
\text { dificultad para cambiar el foco de acción. }\end{array}$ \\
\hline $\begin{array}{l}\text { Grado } 2 \\
\text { "Necesita ayuda } \\
\text { notable" }\end{array}$ & $\begin{array}{l}\text { Deficiencias notables de las aptitudes de } \\
\text { comunicación social verbal y no verbal; problemas } \\
\text { sociales aparentes incluso con ayuda in situ; inicio } \\
\text { limitado de interacciones sociales; y reducción de } \\
\text { respuesta o respuestas no normales a la apertura } \\
\text { social de otras personas. }\end{array}$ & $\begin{array}{l}\text { La inflexibilidad de comportamiento, la } \\
\text { dificultad de hacer frente a los cambios } \\
\text { u otros comportamientos restringidos/ } \\
\text { repetitivos aparecen con frecuencia. } \\
\text { Ansiedad y/o dificultad para cambiar el } \\
\text { foco de acción. }\end{array}$ \\
\hline $\begin{array}{l}\text { Grado } 1 \\
\text { "Necesita ayuda" }\end{array}$ & $\begin{array}{l}\text { Sin ayuda in situ, las deficiencias en la } \\
\text { comunicación social causan problemas } \\
\text { importantes. Dificultad para iniciar interacciones } \\
\text { sociales y ejemplos claros de respuestas atípicas } \\
\text { o insatisfactorias a la apertura social de otras } \\
\text { personas. Puede parecer que tiene poco interés en } \\
\text { las interacciones sociales. }\end{array}$ & $\begin{array}{l}\text { La inflexibilidad de comportamiento } \\
\text { causa una interferencia significativa. } \\
\text { Dificultad para alternar actividades. } \\
\text { Los problemas de organización y de } \\
\text { planificación dificultan la autonomía. }\end{array}$ \\
\hline
\end{tabular}

Fuente: Manual Diagnóstico y Estadístico de los Trastornos Mentales $5^{\text {ta }}$ edición, DSM $5^{(10)}$

Tabla 2. Señales de alerta inmediatas el Trastorno del Espectro del Autismo

\begin{tabular}{ll}
\hline Edad & Señales de alarma \\
\hline 12 meses & No balbucea, no hace gestos (señalar, decir adiós con la mano). \\
18 meses & No dice palabras sencillas. \\
24 meses & No dice frases espontáneas de dos palabras (no ecolálicas). \\
& Cualquier pérdida de habilidades del lenguaje o a nivel social a cual- \\
No específica & quier edad. \\
\hline $\begin{array}{l}\text { Programa de actividades de detección precoz de problemas de salud entre los 0 y 14 años: Coste efectividad de las diferentes } \\
\text { alternativas existentes en la detección precoz de los trastornos del espectro autista (TEA). Madrid: Ministerio de Sanidad, }\end{array}$
\end{tabular}

vida, facilitando el diagnóstico precoz y el desarrollo de diferentes alternativas para el cribado temprano y estrategias en el primer nivel de atención a fin de asegurar una intervención pronta e intensiva $(5,12,13)$. La Organización Panamericana de la Salud (OPS), describe en el Manual para la Vigilancia del Desarrollo Infantil los hitos del desarrollo según la edad cronológica (14) en el contexto de la Metodología de Atención Integrada de las Enfermedades Prevalentes de la Infancia (AIEPI), para dotar de conocimientos y 
Tabla 3. Compendio de señales de alerta del Trastorno del Espectro del Autismo, según edad

\begin{tabular}{ll}
\hline Edad & Signos de alarma \\
\hline Antes de los & $\begin{array}{l}\text { Poca frecuencia del uso de la mirada dirigida a personas. No muestra anticipación cuando va a ser cogido. Falta de interés } \\
12 \text { meses }\end{array}$ \\
$\begin{array}{l}\text { en juegos interactivos simples como el "cucu-tras" o el "toma y daca". Falta de sonrisa social. Falta de ansiedad ante los } \\
\text { extraños sobre los } 9 \text { meses. }\end{array}$
\end{tabular}

Después de Menor contacto ocular. No responde a su nombre. No señala para "pedir algo" (protoimperativo). No muestra objetos. los 12 meses Respuesta inusual ante estímulos auditivos. Falta de interés en juegos interactivos simples como el "cucu-tras" o el "toma y daca". No mira hacia donde otros señalan. Ausencia de imitación espontánea. Ausencia de balbuceo social/comunicativo como si conversara con el adulto.

Entre 18 a 24 No señala con el dedo para "compartir un interés" (protodeclarativo). Dificultades para seguir la mirada del adulto. No meses mirar hacia donde otros señalan. Retraso en el desarrollo del lenguaje comprensivo y/o expresivo. Falta de juego funcional con juguetes o presencia de formas repetitivas de juego con objetos ej. Alinear, abrir y cerrar, encender y apagar, etc.). Ausencia de juego simbólico. Falta de interés en otros niños o hermanos. No suele mostrar objetos. No responde cuando se le llama. No imita ni repite gestos o acciones que otros hacer (ej. muecas, aplaudir). Pocas expresiones para compartir afecto positivo. Antes usaba palabras pero ahora no (regresión en el lenguaje). Pierde habilidades que había adquirido.

\section{Comunicación}

Ausencia o retraso en el lenguaje o déficit en el desarrollo del lenguaje no compensado por otros modos de comunicación.

Edad Uso estereotipado o repetitivo del lenguaje como ecolalia o referirse a sí mismo en $2^{\mathrm{a}}$ o $3^{\mathrm{a}}$ persona. Entonación anormal.

mayores de Pobre respuesta a su nombre. Déficit en la comunicación no verbal (ej. no señalar y dificultad para compartir un "foco de 36 meses atención" con la mirada). Fracaso en la sonrisa social para compartir placer y responder a la sonrisa de los otros. Consigue cosas por sí mismo, sin pedirlas. Antes usaba palabras, pero ahora no. Ausencia de juegos de representación o imitación social variados y apropiados al nivel de desarrollo. Alteraciones sociales

Imitación limitada (ej. Aplaudir) o ausencia de acciones con juguetes o con otros objetos. No "muestra" objetos a los demás. Falta de interés o acercamientos extraños a los niños de su edad. Escaso reconocimiento o respuesta a la felicidad o tristeza de otras personas. No se une a otros en juegos de imaginación compartidos. Fracaso a la hora de iniciar juegos simples con otros o participar en juegos sociales sencillos. Preferencia por actividades solitarias. Relaciones extrañas con adultos desde una excesiva intensidad a una llamativa indiferencia. Escasa utilización social de la mirada.

\section{Alteraciones de los intereses, actividades y conductas}

Insistencia en rutinas y/o resistencia a los cambios en situaciones poco estructurada. Juegos repetitivos con juguetes (ej. Alinear objetos, encender y apagar luces, etc). Apego inusual a algún juguete u objeto que siempre lleva consigo que interfiere en su vida cotidiana. Hipersensibilidad a los sonidos, al tacto y ciertas texturas. Respuesta inusual al dolor. Respuesta inusual ante estímulos sensoriales (auditivos, olfativos, visuales, táctiles y del gusto). Patrones posturales extraños como andar de puntillas. Estereotipias o manierismos motores

\section{Alteraciones de la comunicación}

Desarrollo deficiente del lenguaje, que incluye mutismo, entonación rara o inapropiada, ecolalia, vocabulario inusual para su edad o grupo social.En los casos en que no hay deficiencias en el desarrollo del lenguaje existe uso limitado del lenguaje para comunicarse y tendencia a hablar espontáneamente sólo sobre temas específicos de su interés (lenguaje fluido pero poco adecuado al contexto)

\section{Alteraciones sociales}

Mayores de 5 Dificultad para unirse al juego de los otros niños o intentos inapropiados de jugar conjuntamente. Limitada habilidad para años apreciar las normas culturales (en el vestir, estilo del habla, intereses, etc.). Los estímulos sociales le producen confusión o desagrado. Relación con adultos inapropiada (demasiado intensa o inexistente). Muestra reacciones extremas ante la invasión de su espacio personal o mental (resistencia intensa cuando se le presiona con consignas distintas a su foco de interés).

\section{Limitación de intereses, actividades y conductas}

Ausencia de flexibilidad y juego imaginativo cooperativo, aunque suela crear solo/a ciertos escenarios imaginarios (copiados de los vídeos o dibujos animados). Dificultad de organización en espacios poco estructurados. Falta de habilidad para desenvolverse en los cambios o situaciones poco estructuradas, incluso en aquellas en las que los niños disfrutan como excursiones del colegio, cuando falta una profesora, etc. Acumula datos sobre ciertos temas de su interés de forma restrictiva y estereotipada.

\section{Otros rasgos}

Perfil inusual de habilidades y puntos débiles (por ejemplo, habilidades sociales y motoras escasamente desarrolladas, torpeza motora gruesa). Perfil inusual de habilidades y puntos débiles (por ejemplo, habilidades sociales y motoras escasamente desarrolladas, torpeza motora gruesa). Perfil inusual de habilidades y puntos débiles (por ejemplo, habilidades sociales y motoras escasamente desarrolladas, torpeza motora gruesa). Perfil inusual de habilidades y puntos débiles (por ejemplo, habilidades sociales y motoras escasamente desarrolladas, torpeza motora gruesa) .Perfil inusual de habilidades y puntos débiles (por ejemplo, habilidades sociales y motoras escasamente desarrolladas, torpeza motora gruesa).El conocimiento general, la lectura o el vocabulario pueden estar por encima. Cualquier historia significativa de pérdida de habilidades de la edad cronológica o mental. Ciertas áreas de conocimientos pueden estar especialmente desarrolladas, mostrando habilidades sorprendentes en áreas como matemáticas, mecánica, música, pintura, escultura.

Fuente: Programa de actividades de detección precoz de problemas de salud entre los 0 y 14 años: Coste efectividad de las diferentes alternativas existentes en la detección precoz de los trastornos del espectro autista (TEA). Madrid: Ministerio de Sanidad, Servicios Sociales e Igualdad, $2016^{(15)}$. 
estrategias al personal del primer nivel de atención y recomienda empoderar a los padres en la vigilancia del desarrollo de sus niños (15).

Algunos países de Latinoamérica han desarrollado guías de signos de alarma de alteraciones del desarrollo validadas por recomendaciones de expertos con el objetivo de identificar precozmente el TEA. En Colombia, se utiliza la Tabla de Indicadores de Riesgo desde el nacimiento hasta los 18 años, aplicada a nivel comunitario por cuidadores, educadores, psicólogos, auxiliares de salud pública, entre otros, y en las consultas de programas como crecimiento y desarrollo por personal de salud (8). Argentina, en la primera línea de vigilancia del desarrollo esperado en el primer nivel de atención, hace uso del Instrumento Observación del Desarrollo Infantil (IODI) en niños menores de 4 años (16). Ecuador, en el control de niño sano realiza un tamizaje de rutina denominado Vigilancia evolutiva del neurodesarrollo que comprende desde el nacimiento hasta menores de 9 años (5). Chile cuenta con un instrumento denominado Pautas de cotejo de señales de alerta de TEA que se utiliza luego de identificar señales de alarma con la aplicación de otras escalas de desarrollo psicomotor como, el Test de Desarrollo Psicomotor (TEPSI) y la Escala de Desarrollo Psicomotor (EEDP), realizado en los controles de niño sano (17).

El Perú cuenta con el Test Peruano de Evaluación del Desarrollo del Niño (TPED) que forma parte de la estrategia para identificar signos de alerta del desarrollo, y se aplica como parte del programa de Control de Crecimiento y Desarrollo del niño menor de cinco años (CRED) del primer nivel de atención, aunque no representa una herramienta específica del diagnóstico del TEA (18).

Por lo expuesto, observamos que en Latinoamérica se viene impulsando la detección temprana de señales de alerta del desarrollo en el primer nivel de atención de salud, a través de guías, protocolos o normas técnicas actualizadas o en proceso, para la derivación oportuna al segundo y tercer nivel de atención de salud (19).

\section{Herramientas de ayuda diagnóstica del TEA}

Las herramientas de detección y de ayuda diagnóstica para el cribado del TEA son diversos, siendo las más utilizadas, Modified Checklist for Autism in Toddlers - Revised (M-CHAT), Autism
Diagnostic Interview Revised (ADI-R), y el Autism Diagnostic Observation Schedule (ADOS).

El M-CHAT, se aplica desde los 16 a 30 meses de edad (20) y está diseñada para detectar posibles signos tempranos del TEA o retraso en el desarrollo y cuenta con algunas adaptaciones cuyos niveles de sensibilidad y especificidad son mayores.

La Lista de Verificación Modificada para el Autismo en Niños Pequeños con Seguimiento (M-CHAT/F) está conformada por 23 ítems y dirigida a los padres, consta de una entrevista inicial y otra de seguimiento diseñada para reducir los falsos positivos. La sensibilidad y especificidad del M-CHAT/F es del $38,8 \%$ y $94,9 \%$ respectivamente. El valor predictivo positivo (VPP) fue del $14,6 \%$ y el valor predictivo negativo (VPN) del 98,6\%. (19), estos resultados varían de acuerdo con los estudios de validez que realizan los países de la región $(17,21,22,23)$.

La Lista de Verificación Modificada para el Autismo en Niños Pequeños Revisada con Seguimiento (M-CHAT - R /F) introdujo nuevos criterios de puntuación, además señala que al registrar 8 puntos en la entrevista inicial ya no es necesario realizar la de seguimiento, siendo inmediatamente derivado para la evaluación por el especialista (24).

ADI-R es una entrevista clínica de detección del TEA que recoge información del comportamiento de los niños a partir de entrevistas con los padres o cuidadores. Consta de 93 ítems que exploran tres áreas: lenguaje/comunicación, interacciones sociales recíprocas y conductas e intereses restringidos, repetitivos y estereotipados (25).

El ADOS-2, es una escala estandarizada semiestructurada de evaluación diagnóstica del TEA realizada por un profesional especializado para observar el comportamiento del niño en tareas específicas desde los 12 meses hasta la edad adulta con o sin lenguaje y consta de 5 módulos, cada uno dirigido a una población diferente: comunicación, interacción social, juego o uso imaginativo de los materiales y las conductas restrictivas y repetitivas (25).

Randall et al., compararon ADI-R y ADOS para determinar cuáles de las herramientas utilizadas con mayor frecuencia son más exactas para diagnosticar TEA, identificando que esta última obtuvo mayor sensibilidad, pero en especificidad fue similar a la prueba de ADI-R (25). 
Entre las nuevas herramientas de diagnóstico tenemos la publicada en el estudio de Jensen et al., en colaboración con el Grupo de Estudio del Autismo en el Perú (26), titulado "Detección de autismo en niños mediante el uso combinado de la preferencia de mirada y el M-CHAT-R (GP-M-CHAT/R) en entornos de escasos recursos" realizado en-niños peruanos de 36 a 99 meses. Se les presentaron videos de un minuto de exposición, con escenas abstractas a la derecha de la pantalla y escenas sociales a la izquierda, demostrando que la preferencia visual, tanto el enfoque social como el tiempo de distracción, sirven como marcadores para detectar el riesgo del TEA en los niños. Las diferencias son detectables en tan solo quince segundos de preferencia de mirada. Esta prueba asociada al M-CHAT/R mejora el grado de confiabilidad, de sensibilidad y especificidad para detección temprana del TEA en poblaciones con recursos limitados (26).

\section{Adaptándonos a la nueva realidad: Telesalud}

Diversos países que buscaban ampliar su cobertura de Salud en los sectores rurales, venían investigando y desarrollando el servicio de Telesalud para la Detección, Diagnóstico y Tratamiento de enfermedades comunes y de mayor prevalencia (27). Se ha emitido dispositivos legales para implementar la Detección, Diagnóstico y Tratamiento de TEA (Ley $\mathrm{N}^{\circ} 30150$ ) y de Telesalud (Ley $\mathrm{N}^{\circ} 30421$ ).

En una revisión sistemática en julio 2020, publicada en PLOS ONE, titulada "Uso de Telesalud para facilitar la evaluación diagnóstica del Trastorno del Espectro Autista" (27), se realizó una búsqueda de estudios desde el año 2000 al 2019, identificando el uso del método en tiempo real como la videoconferencia (síncrona), y el método de almacenamiento y reenvío como la Evaluación Diagnóstica de Observación Naturalista (NODA) (asíncrona). Con relación a la precisión diagnóstica, el método de videoconferencia tuvo una alta satisfacción e incluso en algunos estudios no encontraron diferencias significativas entre la forma virtual y presencial. El método NODA, donde los padres o cuidadores grababan videos para ser revisados por los especialistas, alcanzó una sensibilidad de $84,5 \%$ y especificidad del $94,4 \%$. Asimismo, se redujo sustancialmente el tiempo de espera para la evaluación, el diagnóstico y los costos; demostró que es accesible y aceptado por los padres o cuidadores. El estudio concluyó que el uso de Telesalud es aceptable para el diagnóstico del TEA para las familias y los médicos, y merecen más estudios (27).
Otro estudio de revisión sistemática publicada en 2021 sobre la "Implementación de servicios de Telesalud para evaluar, monitorear y tratar los trastornos del Neurodesarrollo", se centró en estudios de viabilidad y aceptabilidad de ta Telesalud para ayudar en el diagnóstico, el seguimiento y el tratamiento de los trastornos del neurodesarrollo antes de la pandemia de COVID-19. El estudio identificó 20925 artículos, siendo 42 los que cumplieron los criterios de inclusión, el mayor número de estudios se basó en las condiciones del TEA y del Trastorno por Déficit de Atención con Hiperactividad (TDAH). Concluyendo que ya existía evidencia sobre Telesalud para el diagnóstico y tratamiento de personas con trastornos del neurodesarrollo, principalmente TEA y TDAH antes de la pandemia COVID-19, con óptimos resultados clínicos y reducción de costos; sin embargo, se requiere más investigación para corroborar esta evidencia (28).

\section{CONCLUSIONES}

Es evidente un incremento en la prevalencia de personas con la condición del TEA. En Latinoamérica se están desarrollando programas para la vigilancia de señales de alerta del neurodesarrollo a fin de realizar diagnóstico y tratamiento oportuno. Se cuenta con diversos cuestionarios y escalas utilizadas a nivel mundial que tienen un alto grado de confiabilidad. Es constante la búsqueda de nuevas formas de ayuda diagnóstica que pueda ampliar la accesibilidad, la reducción de costos y tiempo de espera para el diagnóstico del TEA. La aplicación del método GPM-CHAT/R, contribuye a la detección precoz del TEA principalmente en poblaciones de bajos recursos. La Telesalud con su metodología sincrónica y asincrónica, demuestra ser un nuevo medio viable, accesible, aceptado y confiable para continuar brindando los servicios de salud en pacientes con TEA.

\section{Correspondencia}

Myriam Velarde Incháustegui.

Av. Tomás Marsano 2699, Surquillo, Lima, Perú.

Correo electrónico: myriamvelardei@gmail.com; mvelardei@unmsm.edu.pe

\section{REFERENCIAS BIBLIOGRAFICAS}

1. Centers for Disease Control and Prevention. Key Findings from the ADDM Network. Atlanta: Centers for Disease Control and Prevention; 2014. (Citado el 20 de junio del 2020) Disponible en: https://www.cdc. 
Diagnóstico de Trastorno del Espectro Autista-TEA, adaptándonos a la nueva realidad, Telesalud.

gov/ncbddd/spanish/autism/addm-communityreport/key-findings.html.

2. Centers for Disease Control and Prevention. Prevalence of autism spectrum disorder among children aged 8 years - autism and, developmental disabilities monitoring Network ,11 Sites, United States, 2016. Atlanta: Centers for Disease Control and Prevention; 2020. (Citado el 20 de junio del 2020) Disponible en: www.cdc.gov/ncbddd/autism/ addm.html.

3. Zavaleta $P$, Hernández A, Medina ME. Infraestructura disponible para la atención de los trastornos del espectro autista en el Sistema Nacional de Salud. Informe Final. Ciudad de México: Secretaría de Salud, Comisión Coordinadora de Institutos Nacionales de Salud y Hospitales de Alta Especialidad, Instituto Nacional de Psiquiatría Ramón de la Fuente Muñiz; 2018.

4. Ministerio de Salud y Protección Social. Protocolo Clínico para el Diagnóstico, Tratamiento y Ruta de Atención Integral de Niños y Niñas con Trastorno del Espectro Autista. Guía Técnica. Bogotá: Instituto de Evaluación Tecnológica en Salud, Ministerio de Salud y Protección Social; 2015.

5. Ministerio de Salud Pública. Trastornos del espectro autista en niños y adolescentes: detección, diagnóstico, tratamiento, rehabilitación y seguimiento. Guía de Práctica Clínica. Quito: Ministerio de Salud Pública, Dirección Nacional; 2017. (Citado el 20 de junio del 2020) Disponible en: https://www.salud.gob.ec/wpcontent/ uploads/2014/05/GPC_Trastornos_del_espectro_ autista_en_ninos_y_adolescentes-1.pdf.

6. Ministerio de la Mujer y Poblaciones Vulnerables. Plan Nacional para las Personas con Trastorno del Espectro Autista. Norma Técnica. Lima: Ministerio de la Mujer y Poblaciones Vulnerables, Consejo Nacional para la Integración de la Persona con Discapacidad; 2021.

7. Organización Mundial de la Salud. Trastornos del espectro autista. Ginebra: Organización Mundial de la Salud; 2019. (Citado el 20 de junio del 2020) Disponible en: https://www.who.int/es/news-room/ fact-sheets/detail/autism-spectrum-disorders.

8. Ministerio de Salud y Protección Social. Protocolo clínico para el diagnóstico, tratamiento y ruta de atención integral de niños y niñas con trastorno del espectro autista. Guía Técnica. Bogotá: Instituto de Evaluación Tecnológica en Salud, Ministerio de Salud y Protección Social; 2015.

9. Ministerio de Salud y Desarrollo Social. Consenso sobre diagnóstico y tratamiento de personas con trastorno del espectro autista. Gráfico. Buenos Aires: Ministerio de Salud y Desarrollo Social, Dirección Nacional de Calidad en Servicios de Salud y Regulación Sanitaria; 2019.
10. American Psychiatric Association. Diagnostic and Statistical Manual of Mental Disorders (DSM-5). Quinta edición ed. VA, editor. Arlington: American Psychiatric Publishing; 2013.

11. World Health Organization. International Statistical Classification of Diseases and Related Health Problems. 10th ed. Ginebra: World Health Organization; 1992.

12. Rojas V, Rivera A, Nilo N. Actualización en diagnóstico e intervención temprana del trastorno del espectro autista. Revista Chilena de Pediatría. 2019 Agosto; 90(5): p. 478-484.

13. Carrascón C. Señales de alerta de los trastornos del espectro autista. En: Primaria AEdPdA. $15^{\circ}$ Curso de Actualización de Pediatría. Madrid: Lua Ediciones 3.0 SL.; 2018. p. 331-334.

14. Vicente M, Paredes I, Gavín P, Martín J. Programa de actividades de detección precoz de problemas de salud entre los 0 y 14 años. Coste - efectividad de las diferentes alternativas existentes en la detección precoz de los trastornos del espectro autista (TEA). Informes de Evaluación Técnicas Sanitarias: IACS. Madrid: Ministerio de Sanidad.

15. Figueiras AC, Neves de Souza IC, Ríos VG, Benguigui Y. Manual para la Vigilancia del Desarrollo Infantil (0 a 6 años) en el Contexto del AIEPI. Washington DC: OPS; 2011.

16. Ministerio de Salud. Criterios para la aplicación del instrumento de observación del desarrollo infantilGuía. Buenos Aires: Secretaría de Promoción de la Salud, Ministerio de Salud; 2017.

17. Ministerio de Salud. Guía de Práctica Clínica: Detección y Diagnóstico Oportuno de los Trastornos del Espectro Autista (TEA). Santiago: Ministerio de Salud, Departamento de Discapacidad y Rehabilitación; 2011.

18. Ministerio de Salud. Norma Técnica para el Control del Crecimiento y Desarrollo de la Niña y el Niño menor de cinco años. Norma Técnica. Lima: Ministerio de Salud; 2017.

19. Instituto Nacional de Salud. Control de crecimiento y desarrollo de los niños Menores de 12 años. Series revisiones ultra rápidas $\mathrm{N}^{\circ}$ 09-16. Lima: Instituto Nacional de Salud, Unidad de Análisis y Generación de Evidencias; 2016.

20. Petrocchi S, Levante AL, Lecciso F. Systematic Review of Level 1 and Level 2 Screening Tools for Autism Spectrum Disorders in Toddlers. Brain Sci. 2020; 10(3): 180. doi: 10.3390/brainsci10030180

21. Ministerio de Salud y Protección Social. Protocolo clínico para el diagnóstico, tratamiento y ruta de atención integral de niños y niñas con trastorno del espectro autista. Bogotá: Ministerio de Salud y Protección Social; 2015. (Citado el 20 de junio del 2020) Disponible en: https://www.minsalud.gov. co/sites/rid/Lists/BibliotecaDigital/RIDE/DE/CA/ 
Protocolo-TEA-final.pdf.

22. Coelho-Medeiros ME, Bronstein J, Aedo K, Pereira $\mathrm{JA}$, Arraño V, Perez CA, et al. Validación del M-CHAT-R/F como instrumento de tamizaje para detección precoz en niños con trastorno del espectro autista. Revista Chilena de Pediatría. 2019; 90(5): 492-499.

23. Guthrie W, Wallis K, Bennett A, Brooks E, Dudley J, Gerdes $M$, et al. Accuracy of Autism Screening in a Large Pediatric Network. Pediatrics. 2019;144(4):e20183963. doi: 10.1542/peds.20183963.

24. Robins D. Validation of the modified checklist for Autism in toddlers, revised with follow-up (M-CHATR/F). Pediatrics. 2014;133(1):37-45. doi: 10.1542/ peds.2013-1813

25. Randall M, Egberts KJ, Samtani A, Scholten R, Hooft L, Livingstone N, et al. Diagnostic tests for autism spectrum disorder (ASD) in preschool children. Cochrane Database of Systematic Reviews 2018; 7: CD009044. DOI: 10.1002/14651858.CD009044. pub2
26. Jensen K, Noazin S, Bitterfeld L, Carcelen A, Vargas-Cuentas N, Hidalgo D, et al. Autism Detection in Children by Combined Use of Gaze Preference and the M-CHAT-R in a Resource-Scarce Setting. J Autism Dev Disord. 2021;51(3):994-1006. doi: 10.1007/s10803-021-04878-0

27. Alfuraydan M, Croxall J, Hurt L, Kerr M, Brophy S. Use of telehealth for facilitating the diagnostic assessment of autism spectrum disorder (ASD): A scoping review. PLoS ONE. 2020; 15(7): e0236415. DOI: 10.1371/journal.pone.0236415

28. Valentine A, Hall S, Young E, Brown B, Groom M, Hollis C, et al. Implementation of Telehealth Services to Assess, Monitor, and Treat Neurodevelopmental Disorders: Systematic Review. J Med Internet Res. 2021;23(1):e22619. doi: 10.2196/22619

Recibido: $12 / 04 / 2021$ Aceptado: 03/08/2021 